Session 3470

\title{
Diversity: An Engineering Process
}

\author{
Andre H. Sayles \\ United States Military Academy \\ West Point, NY 10996
}

\begin{abstract}
Engineers often prefer to work with processes, particularly those that allow for assumptions, inputs, and outputs. Unfortunately, engineers seldom pursue diversity management as perhaps a secondary career field. In this paper, organizational diversity is represented by an engineeringlike process having three primary phases and a supporting phase. The Leading Diversity Process Model (LDPM) is described by a modified pyramid with four parts defined as accepting differences, understanding differences, valuing differences, and role models. These same terms are often used in diversity conversations and literature; however, the current success story is derived from the manner in which the four components are defined and linked together to form a process. Like most engineering processes, the diversity model is receptive to creativity and innovation, along with standard problem-solving methodologies. Since July 2002, the LDPM has been presented to diverse audiences, including students, faculty, military leaders, and equal opportunity professionals. This paper offers an abbreviated discussion of the evolving description of each part of the model, followed by a brief summary of audience reaction to date.
\end{abstract}

Introduction

Today, organizations and institutions continue to seek out creative ways to meet diversity goals and, in some cases, compete for small populations within particular ethnic and gender groups. However, innovative approaches to meeting diversity challenges have not included the notion that organizational diversity can be viewed as an engineering-like process. Instead, experts tend to focus only on particular aspects of diversity. Sociologists offer insights into the impact of equality and opportunity on various cultural groups in society. Linguists and psychologists have explained communication differences across ethnic and gender groups and the impact of those differences on work, school, and social environments. Historians describe how different groups have been treated over the years and how their contributions have shaped our nation. Diversity consultants offer analyses of different cultures and suggest the types of work environments that may or may not be appealing based on gender or ethnicity. Various segments of our population sponsor observances and other productions that promote both recognition and understanding of particular cultures. What is missing among all of these efforts is a process that offers a methodology for selecting, implementing, and assessing various diversity actions, activities, and programs.

Proceedings of the 2004 American Society for Engineering Education Annual Conference \& Exposition Copyright (C) 2004, American Society for Engineering Education 
For over 200 years, engineers have solved some of the world's toughest problems. Many of these solutions are products of an engineering thought process, design process, or problem solving methodology. Organizational diversity can be viewed as a process similar to the methods through which engineers solve technical problems. Over the past two years, a novel approach to diversity, based on organizational effectiveness, has enabled many people from various backgrounds and levels of experience to better understand how diversity works.

\section{Leading Diversity Process Model—The Concept}

The Leading Diversity Process Model (LDPM) is very simple. In fact, the simplicity is what makes it work for different audiences. The LDPM shown in Figure 1 has the three primary components of accept differences, understand differences, and value differences, while the role model section at the top is external to the primary process, but part of the overall concept of enhancing organizational effectiveness through diversity. The core thread in the model is how differences in people are handled by individuals and organizations or institutions.

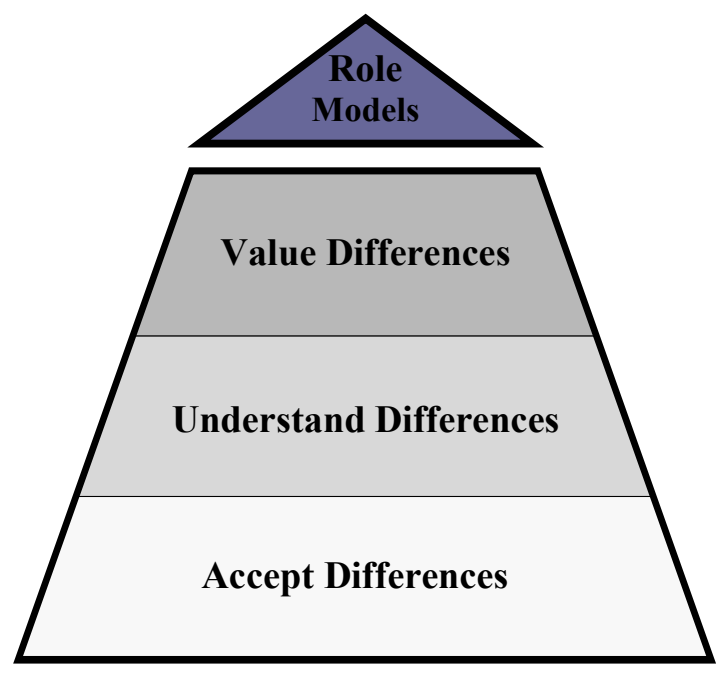

Figure 1. Leading Diversity Process Model

Diversity has been defined many different ways, ranging from broad to narrow, depending on the context. The definition of diversity associated with the LDPM is differences in people that can have an impact on the effectiveness of an organization. The impact can be either positive or negative, depending on the organization, the nature of the difference, and how the difference is perceived. Differences come in many forms, are derived from many sources, and are reflected in language, behavior patterns, thought processes, biases, beliefs, preferences, and perceptions. It is important to have a means of understanding such differences in people, knowing how those differences affect the organization, knowing when and how to promote certain differences and suppress others, and ultimately having the ability to use that knowledge to achieve enhanced organizational effectiveness. These accomplishments describe the concept of Leading Diversity, a thought process that goes beyond common practices of managing or leveraging diversity. 
The Leading Diversity Process is simplified through assumptions in the same manner that an engineer might narrow the scope of a problem until the challenge at hand matches the potential of available resources. Since a perfect solution to organizational diversity will never be found, the LDPM need not be perfect in order to be effective. In fact, a marginally effective LDPM would likely be as good as any other documented diversity process. Assumptions are necessary to maintain the right focus when addressing diversity issues, while avoiding the many pitfalls associated with precisely defining terms or trying to solve every issue in a single pass. Perhaps the most important simplifying assumption in the Leading Diversity Process is that culture, when defined broadly, is not a stand-alone difference to be accounted for. Instead, culture is treated as the basis for all key differences to be addressed by the process. For example, gender-based conversation styles are assumed to derive from differences in male and female childhood social factors and are generalized as cross-cultural communications. ${ }^{1}$ Likewise, ethnic, religious, and other differences are treated as cultural factors. The model input is the individual difference to be considered, leaving culture as simply an overarching basis for those inputs.

Examples of typical LDPM differences are shown in Table 1. Because race does not always align with specific cultural factors, it is not a preferred LDPM input. Ethnicity is favored over race. In pursuing diversity as a process, a good practice is to focus on differences according to the known or anticipated impact on the specific organization. For example, in many organizations, the key differences may be ethnicity, gender, and religion. Other organizations may have a different set of priorities, some of which may or may not be listed below.

\begin{tabular}{|c|c|c|}
\hline Ethnicity & Age & Disabilities \\
\hline \hline Gender & Marital Status & Work Experiences \\
\hline \hline Religion & Economic Status & Personal Experiences \\
\hline \hline Education & Physical Ability & Sexual Orientation \\
\hline \hline Language & Political Orientation & Organizational Position \\
\hline
\end{tabular}

Table 1. Example Differences That Impact Organizations

An effective approach to the Leading Diversity Process is to start with a narrowed list of differences and expand into other areas as organizational members better understand the process and make progress accordingly. Understanding and valuing some differences normally leads to similar positive consideration for other differences as a by-product. The lack of focus early in a program can dilute a good diversity effort to the point of ineffectiveness. On the other hand, the initial act of having to identify which differences have the greatest impact on the organization requires the top-down thought process necessary for pursuing diversity through the LDPM.

Any number of assumptions may become necessary as diversity goals are pursued through the LDPM. However, before discussing each component of the Leading Diversity Process Model in the next section, the final global assumption is that diversity can bring value to an organization. The validity of this assumption becomes evident over time as LDPM objectives are achieved within an organization. Literature is available for further investigation of this topic. ${ }^{2}$ 


\section{Accept Differences}

After making initial assumptions and thinking about the impact of differences on an organization or institution, the entry point into the LDPM is accepting the notion that differences matter. Those who cannot accept this notion have no basis or motivation to move to the next levelunderstanding differences. A key to an effective diversity program is that the organization is willing to acknowledge and respect certain differences in people while also expecting members to adapt to the organizational culture. This thought process is best explained using Figure 2 .

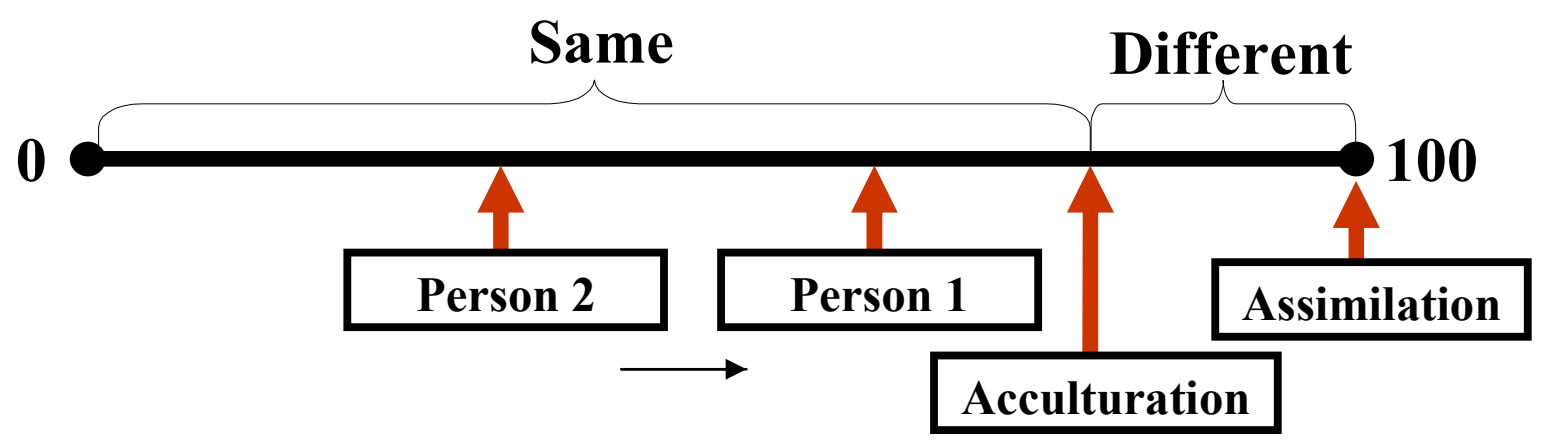

Figure 2. Adapting to the Organizational Culture

At this point it is helpful to first make two additional assumptions. The first assumption is that the organization of concern has an identifiable dominant culture. The second is that the culture derives from organizational leadership over a period of time. An alternative view is that the organizational culture is based on the dominant culture of its members. In any case, it is necessary to establish that dominant behavior patterns exist in order to discuss deviations from those patterns or differences.

When an individual joins an organization, several possibilities exist in terms of acculturation or adapting to the existing culture. Referring to Figure 2, 100\% acculturation is defined as assimilation, the point at which one gives up on his or her past and totally adapts to the existing culture. R. Roosevelt Thomas, Jr. described assimilation as cutting saplings from their roots in the forest and grafting them to a larger tree. Sometimes assimilation is a personal choice; sometimes the work environment leaves no other option. Although not always evident to leaders, most organizations limit effectiveness when assimilation pressures exist.

The amount of effort necessary to adapt to an existing culture depends on the start point, which is determined by the background of the individual when compared to the organizational culture. In Figure 2, Person 1 and Person 2 enter the organization with different backgrounds and will experience different challenges in adapting to the organizational culture to the arbitrarily designated expected level. Person 2 would likely have a difficult time giving up his or her past in order to transition all the way across to the assimilation point. In this scenario, organizational members are considered to be the same to the extent of acculturation, but different to the extent that individual differences are respected by the organization. Sameness is necessary for 
commonality of purpose and predictability, while respecting some differences is necessary for individuality and inspired performance. The "same" portion of Figure 2 is relatively easy to manage, while management of the "different" portion determines an organization's potential for a successful diverse environment and the corresponding enhanced mission effectiveness. The "different" portion of Figure 2 serves as the basis for many diversity issues - from mentorship to the reasons for having special activities for minority population segments. Acknowledgement of ethnic and gender differences implies acceptance of opportunities for individuals to nurture those differences at appropriate times and places. Opportunities to nurture differences, in turn, support the complementary acculturation requirement by inspiring individuals to work harder to adapt to the dominant organizational culture or be the "same" when the time comes to do so. ${ }^{3}$

In theory, an optimal balance between acculturation expectations and respect for differences can lead to improved organizational effectiveness. As suggested for the hypothetical organizations in Figure 3, that balance may be different for each organization. Organization 1 best operates in a "come as you are" environment, while Organization 3 requires extensive acculturation. An example of Organization 3 may be military or law enforcement organizations where safety concerns demand that individual responses be predictable during difficult situations. Otherwise, a life may be lost. Many organizations fall somewhere in the middle like Organization 2. An example of Organization 1 is left to the reader.

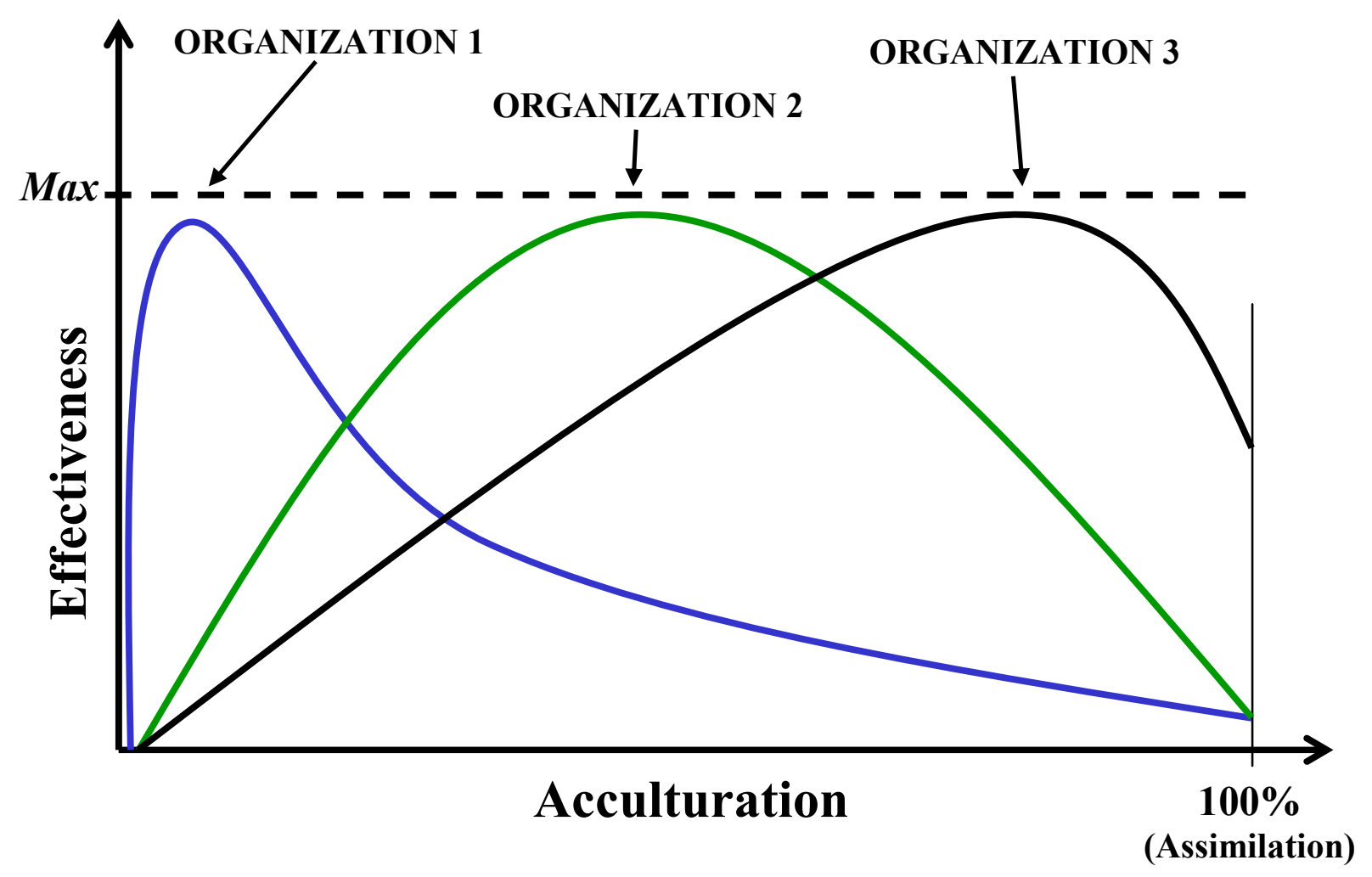

Figure 3. Acculturation versus Diversity Effectiveness for Fictitious Organizations 


\section{Understand Differences}

Accepting the idea that differences matter in an organization should lead to a desire to understand what those differences are and when they matter. Understanding cultural-based differences is the part of the LDPM that requires the most effort. Over time, knowledge of differences continues to improve, but one never reaches the point where growth is no longer possible. An understanding of various cultures reveals the value that those differences can bring to an organization. It becomes evident how diversity can enhance decision-making and organizational effectiveness, and how individuals can be placed in positions that take advantage of the special contributions that can be derived from cultural differences.

Many books have been written about ethnic and gender differences; however, literature is not a substitute for the individual discovery process that is essential to lifelong diversity learning. Understanding differences through discovery originates from a personal commitment to communicate with those who are different. The understand differences section of the LDPM establishes the need for ethnic and other observances that provide concentrated educational opportunities for those who are interested. Many organizations offer other diversity education opportunities such as speeches, lectures, workshops, and activities provided through sponsored cultural groups.

Regardless of the means, understanding differences is a life-long process that may be approximated by a mathematical function. The preferred function is the curve shown in Figure 4 that represents growth through a daily effort to learn about other cultures. At Point A on the graph, the individual remains ignorant of cultural differences, having a tendency to say and do

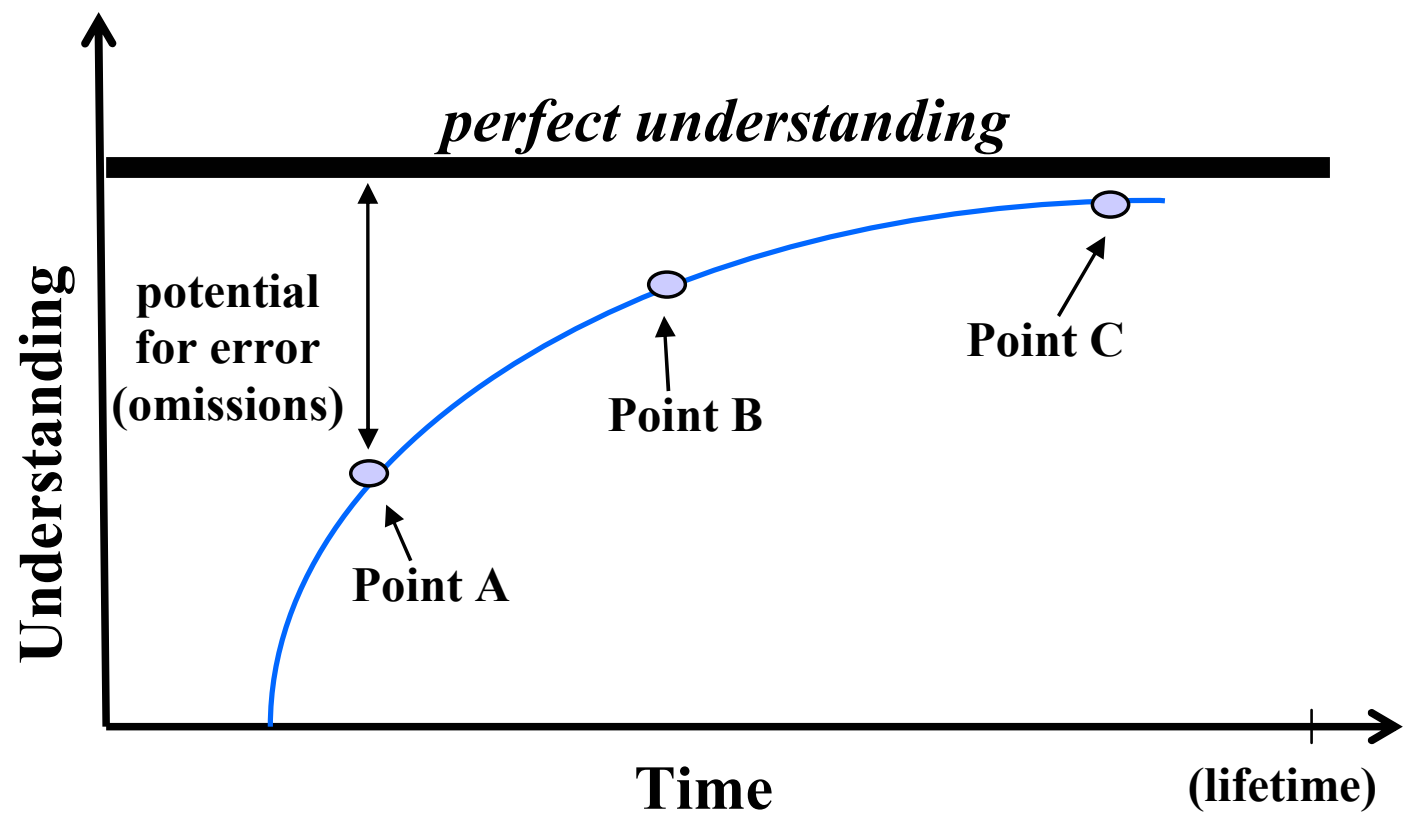

Figure 4. Graphical Representation of Understanding Differences

Proceedings of the 2004 American Society for Engineering Education Annual Conference \& Exposition Copyright (C) 2004, American Society for Engineering Education 
things, often by omission, that are contrary to an effective diverse environment. Point B is an arbitrary location where an individual has a level of understanding of differences such that if an action contrary to a positive environment occurs, the errant choice will soon be realized and corrected. At Point C, the individual is close enough to the "perfect understanding" asymptote to clearly see the valuable contributions that diversity can bring to an organization. The smooth curve suggests a consistent effort to pursue an understanding of the cultures represented in an organization. The progress of those who only learn through periodic observances and similar activities may be better represented by a stair-step type function, assuming all knowledge is retained. A commitment to the daily pursuit of understanding is obviously the best approach to accomplishing the understand differences section of the LDPM.

\section{Value Differences}

An understanding of differences in people eventually reveals the powerful impact that diversity can have on an organization. Although many individuals are willing to readily proclaim their support for diversity, valuing differences can only follow a minimum level of understanding. Otherwise, the argument is that one can value what is not understood. Valuing differences requires a significant investment of effort over a substantial period of time as described in the previous section.

At this level of the LDPM, behaviors and attitudes towards diversity are re-defined. The benefits of diversity become the reasons for diversity. It is increasingly more apparent how diversity can be leveraged to enhance organizational effectiveness. Internally, organizations focus less on raw demographics and more on the potential contributions derived from cultural differences based on individual experiences, including those based on gender, ethnicity, religion, physical limitations, education, work experience, and political orientation. Leaders begin thinking about the need for diverse leadership in a diverse organization. Organizations having a constituency or customer begin to think about the impact of internal diversity on external perceptions. Mission accomplishment becomes more than delivery of a particular product. The manner in which that product is created also matters. When available, the opinions of those whose background differs from the dominant culture are sought. When not available, action is taken to make those opinions available in the future. Ultimately, leaders strive to maximize the positive impact of diversity in as many ways as possible.

\section{Role Models}

Success in the three primary levels of the LDPM will lead to inspired performances throughout the organization by individuals working to their full potential. The presence of role models can aid this process; however, the availability of senior personnel across all cultural domains will not likely occur and should not be viewed as a limiting factor in the three primary stages of the LDPM.

Depiction of the role models section of the LDPM in Figure 1 above and separated from the rest of the pyramid offers several important messages. First, the small gap in the model suggests that 
accept differences, understand differences, and value differences can be achieved separately without the presence of a particular role model. The gap also suggests a time separation. Specifically, the primary impact of role models is on the individual. When application of the LDPM has inspired individuals to work to their full potential, the presence of role models serves to bridge that present situation to the future. Role models represent what that individual can be if he or she continues that inspired performance. The possibility of future success may also lead to an even more inspired performance in the present.

From the above discussion, one can perhaps see value in the availability of role models across ethnic, gender, and other boundaries. Many individuals need to see someone "like them" reach a certain level of success before they know for sure that they can do it. However, this thought process should not be applied universally. For example, some ethnic or gender minorities in a particular environment really do need to see a role model at some point. Other individuals of the same groups may have full confidence in achieving their goals and have no particular need for role models. One could even argue that the need for role models not only depends on the individual, but is also a function of the environment in which that individual is trying to succeed. The bottom line argument supporting the LDPM is that some individuals need role models, while others do not. Where possible, organizations should strive to have representation at all levels.

\section{LDPM Implementation}

One strength of the LDPM is that it can be as simple or as complex as desired. Unlike most diversity efforts, the basic process is intended for all organizational members instead of just leaders. Simplicity makes this possible. Involving an entire organization in the diversity process will lead to outcomes that otherwise could not be achieved. Although the notion of nurturing differences has not been fully developed in this paper, it is a good illustration of the impact of involving the entire organization. Those who differ from the dominant organizational culture value opportunities to return to their roots by interacting with individuals of similar backgrounds. These opportunities often exist through gender or ethnic minority oriented activities or simply several individuals sharing a table in the cafeteria. Excessive nurturing of differences may be perceived as separatism, negatively impacting the organization. Too few opportunities may lead to frustration and inhibit performance, negatively impacting the organization. If all organizational members understand how the LDPM works, then all will understand both the need to nurture differences and the necessary limits. Majority members will understand the need for cultural-based activities, while minority members will understand the impact of excessive isolation.

Although a specific implementation program has not been established, a possible scenario is shown in Table 2. The start point is a general appreciation for the importance of diversity at levels above the organization - international, national, state, and institution as appropriate. Those who live or work in a diverse environment have already thought about diversity to this extent. The next step is to focus on organization-level diversity through the LDPM. A good practice is to offer the process to organizational leaders first. When an appropriate level of "buyin" has been accomplished, then move to the remainder of the organization with leaders present. 
The third step is a framework consisting of policies, procedures, and resources that support LDPM activities. In many organizations, this framework already exists, to include excellent programs and activities. The final step is to make the LDPM the basis of future diversity-related activities, depending on where the organization is perceived to be through initial assessments or observations. For example, the primary purpose of most activities will be to promote understanding of cultural differences. However, the value of such activities will be limited if the argument that differences matter in the organization is not accepted first. Those who do not believe that differences matter have no motivation to participate in activities that promote understanding of those differences. Thus, a preliminary program at the accept differences level would make sense.

\begin{tabular}{|c|l|l|}
\hline 1. & $\begin{array}{l}\text { Importance of Diversity: International, National, State, } \\
\text { Institutional, And Other As Appropriate. }\end{array}$ \\
\hline 2. & $\begin{array}{l}\text { Fluency in the Leading Diversity Process Model (LDPM)_Leaders } \\
\text { First, Then All Others. }\end{array}$ \\
\hline 3. & $\begin{array}{l}\text { Supporting Framework: Policies, Procedures, Guidelines, and } \\
\text { Resources As Appropriate. }\end{array}$ \\
\hline 4. & $\begin{array}{l}\text { LDPM Implementation: Assess Needs, Implement Programs, } \\
\text { Encourage Creativity At All Levels. }\end{array}$ \\
\hline 5. & $\begin{array}{l}\text { Assessment At Individual and Organizational Levels. Plan For } \\
\text { Improvement. }\end{array}$ \\
\hline
\end{tabular}

Table 2. Example LDPM Implementation

Benefits of the LDPM will be revealed in many ways. A common understanding of how diversity works opens the door to conversations that otherwise would never take place. This common understanding also promotes creativity and innovation from individuals who were previously uncomfortable discussing diversity. Finally, organizational members begin to value differences and voluntarily support organizational diversity efforts.

\section{Assessment}

Since the LDPM is a new concept, a formal assessment process is yet to be developed. On the surface, it appears that both organizational and individual assessments can range from simple to complex. The LDPM structure serves as a good reference for self-assessment. Individuals should have a pretty good idea whether or not they pay attention to differences. Likewise, each person should know how much effort has been put into understanding others and the outcomes of that effort. Valuing differences is perhaps a little more difficult for the self-assessor. Many of us already believe that we value differences, but in many cases we do not have adequate knowledge of differences to really know where we stand. Beyond an adequate understanding of individual differences, indicators of the extent to which differences are valued can be the manner in which different points of view are handled, the manner in which diversity is sought, or the ability to talk 
about diversity principles. In Figure 5, several preliminary ideas on assessment are given for each level of the LDPM. The best approach to Figure 5 is to work from bottom to top.

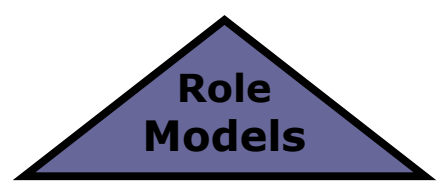

1. Role models available in the organization or otherwise visible?

2. Available role models willing to serve that purpose and understand why?

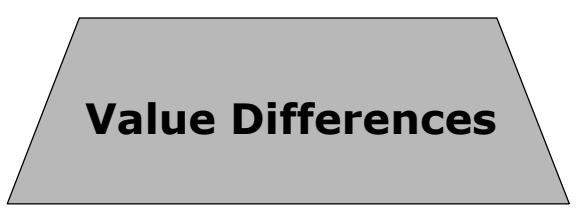

1. Considers diverse opinions in lieu of immediate dismissal of alternative views?

2. Seeks out individuals of different backgrounds when solving tough problems?

3. Believes in or has experienced the power of organizational diversity?

4. Excited about discussing diversity?

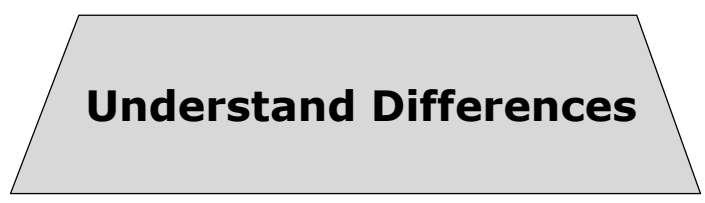

1. Comfortable speaking to or about different groups without fear of making a mistake?

2. Can at least occasionally make immediate adjustments in style and language to inspire others?

3. Seeks out observances and public opportunities to learn about different cultures?

4. Committed to seeking out different individuals for interaction on a daily basis?

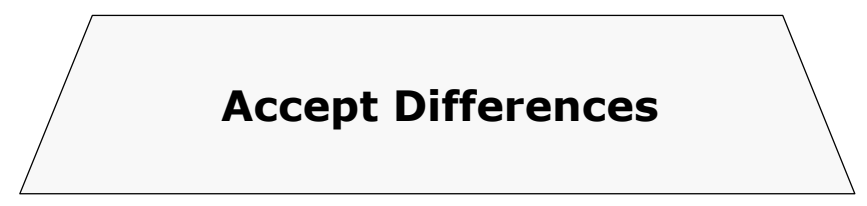

1. Believes that everyone ought not be treated the same all of the time?

2. Believes that opportunities to nurture differences can be good for the organization?

3. Understands the role of different cultures in the organization?

4. Believes that acculturation is necessary, but some differences must be respected?

\section{Figure 5. Sample LDPM Assessment Questions}


Implementation of the strategy in Table 2 and development of organizational assessment tools typically requires a central office or at least an individual who is responsible for organizational diversity. For large organizations, it is essential to have both a high-level, full-time diversity champion and a strategic plan that offers the vision and goals for the future and methodologies for assessing the past (steps 3, 4, and 5 in Table 2). Normally, diversity is best served by individuals not associated with the compliance and complaint responsibilities of equal opportunity professionals. Since these tasks are often short-term, high priority duties, longer term diversity responsibilities will likely become secondary.

\section{Qualitative Results}

This novel approach to organizational diversity has been presented in seminar format to a wide variety of audiences, including students, college staff and faculty members, equal opportunity professionals, human resource managers, and organizational leaders. Most individuals, including those who work as professionals in the field, say that the process brings about a new understanding of diversity. Several have been quoted as saying that despite many years in the equal opportunity profession, they never really understood how diversity works. Often, seminar participants can relate past situations to the model and for the first time understand what happened and why. The LDPM is now being used at the beginning of equal opportunity programs for executives, with follow-on seminars designed to address the different parts of the model.

In an academic environment where diversity was seldom discussed in a casual manner, informal discussions now take place throughout the academic area. One of the most encouraging outcomes has been faculty ability to recall the LDPM and apply it to many different scenarios. In one case, a faculty member interviewing for a position accurately used the model to analyze a situation nearly six months after exposure to the LDPM through a single one-hour seminar. Other faculty members have taken an interest in specific aspects of the model, including the best mathematical description of the lifelong learning process associated with understanding differences. Finally, the linear graph describing the concept of same, but different has been used to analyze situations related to international relations and the role of special purpose educational institutions. Communicating about diversity issues is usually a step in the right direction, regardless of the actual topic.

Although little work has been done so far to develop formal assessment tools for individuals and organizations using the LDPM, quantitative outcomes are expected to match the anecdotal feedback already presented. Three important aspects of the LDPM are its simplicity, the fact that it offers an identifiable process based on individual differences, and the ability to do quick, firstorder self-assessments. Figure 6 offers a sample of quantitative feedback from a group 12 to 18 months after a single, one-hour seminar. Nearly $80 \%$ could recall at least three of the four parts of the model, while two-thirds felt that the Leading Diversity concept changed their outlook on organizational diversity and resulted in a new appreciation of differences in people. Only a small percentage misinterpreted the model. Nearly all misinterpretations are at the bottom level where "accept differences" is believed to be accepting differences of others as opposed to simply accepting the argument that differences matter at all.

Proceedings of the 2004 American Society for Engineering Education Annual Conference \& Exposition Copyright $@$ 2004, American Society for Engineering Education 

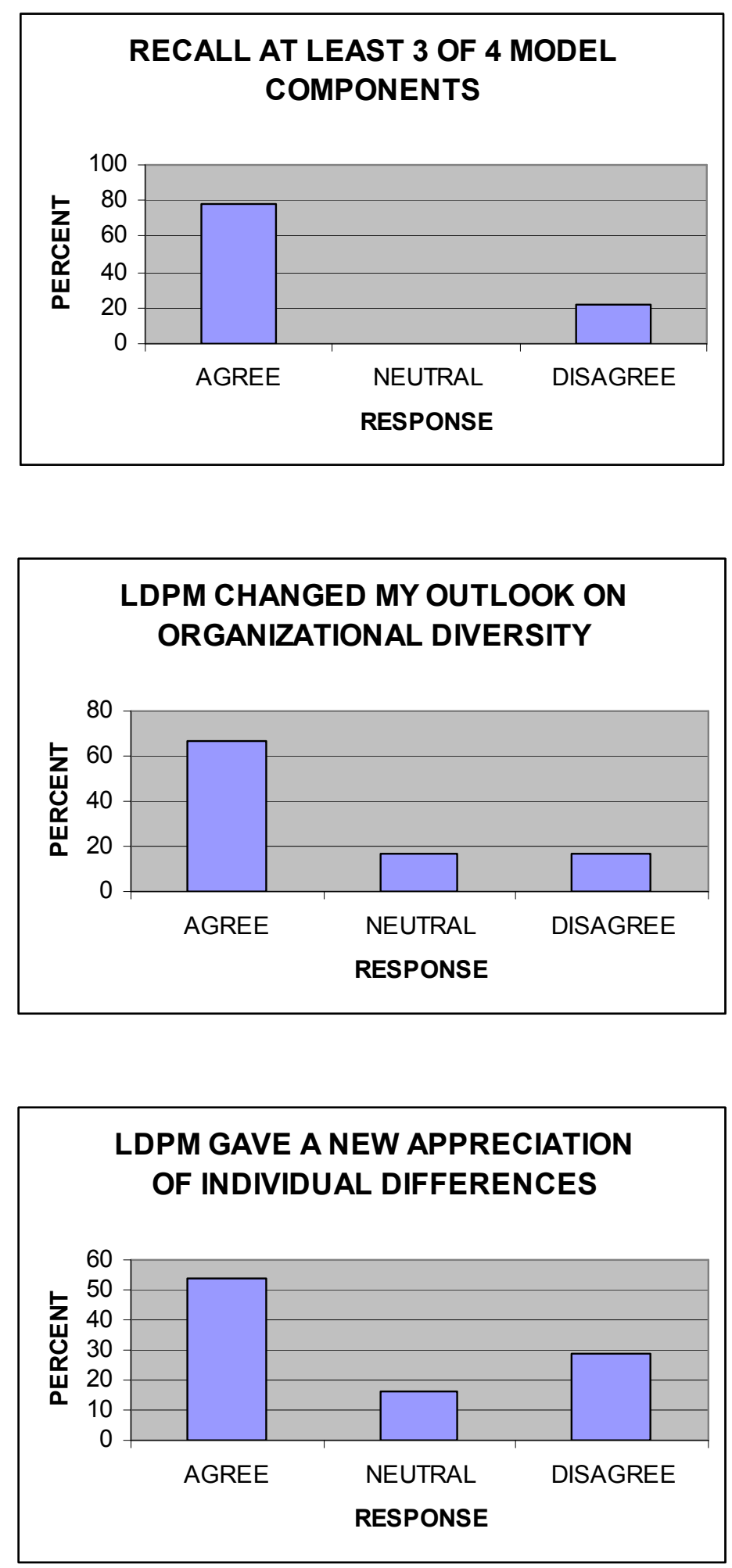

Figure 6. Sample Participant Responses 12-18 Months After LDPM Seminar

Proceedings of the 2004 American Society for Engineering Education Annual Conference \& Exposition Copyright (C) 2004, American Society for Engineering Education 


\section{Summary}

The principle argument in this paper is that the practice of arbitrarily implementing diversity programs and initiatives is no longer the best way to move forward. The simple Leading Diversity Process Model provides a roadmap for implementing programs that address the diversity needs of an organization. The built-in assessment works for both individuals and groups. Following the road map, programs can be designed for the part of the process appropriate for how far the organization has come in accepting, understanding and valuing differences in people.

The LDPM has been presented in many different environments and tested in many different situations. It is capable of not only guiding diversity programs, but also can be used figure out the right course of action in specific situations related to individual differences. In the future, the LDPM will be further developed, documented, and supplemented with descriptive literature and an assessment model. As is the case with most engineering models, weaknesses will be discovered. However, like diversity itself, the LDPM need not be perfect in order to be effective. The LDPM is the result of an interest in diversity that spans 20 years. It is based primarily on personal experience and collaboration with professionals. 
Bibliography

1. Deborah Tannen, You Just Don't Understand: Women and Men in Conversation. New York: William Morrow and Company, Inc., 1990, p. 47.

2. R. Roosevelt Thomas, Jr., Beyond Race and Gender: Unleashing the power of Your Total Work Force by Managing Diversity. New York: AMACOM, 1991.

3. Andre H. Sayles, “On Diversity,” U.S. Army War College Strategic Studies Institute, 1998.

http://carlisle-www.army.mil/ssi/pubs/1998/divrsity/divrsity.htm

Biography

ANDRE H. SAYLES is Professor and Head of the Department of Electrical Engineering and Computer Science at the U.S. Military Academy. He has a B.S. degree from USMA, and a M.S. and Ph.D. in Electrical Engineering from Georgia Tech. He also holds a M.S. in General Management and M.A. in National Security. Along with administrative and teaching responsibilities, he has conducted numerous diversity seminars over the last 3 years.

\section{Acknowledgments}

The author thanks the USMA Leading Diversity Working Group for support of this program. Contributors are Jose Picart, Michelle Nadeau-Schaff, Adam Grijalba, Thomas Jessen, and Mark Johnson. 